\title{
Review of: "“Vaccine-Induced Covid-19 Mimicry" Syndrome:Splice reactions within the SARS-CoV-2 Spike open reading frame result in Spike protein variants that may cause thromboembolic events in patients immunized with vector-based vaccines"
}

\author{
Maurizio Federico ${ }^{1}$ \\ 1 Istituto Superiore di Sanità
}

Potential competing interests: The author(s) declared that no potential competing interests exist.

The authors propose a mechanism through which anti-SARS-CoV-2 vaccination with adenoviral vectorbased preparations triggers, although rare, often fatal thrombotic events (CVST, SVT). Essentially, they sought to demonstrate that the production of forms deleted of the trans-membrane domain of the vaccineproduced Spike protein leads to its release from vector-expressing cells, thereby initiating the severe sideeffects starting from interaction with the ACE-2 cell receptor. The truncated forms of the vaccine Spike protein are supposed to originate from splicing events that adenoviral RNA may recognize by virtue of a number of cryptic donor/acceptor sites identified in optimized sequences of adenoviral vaccine-encoded Spike.

The hypothesis is well formulated and seems quite attractive. Generally speaking, however, the strong antibody response following vaccine doses should be for the most part consequence of presentation in MHC Class II of peptides from cell-free Spike rather than its simple recognition on surface of cells expressing the vaccine product(s). Moreover, large membrane-anchored viral proteins can be easily released by infected cells even in their full-length form, so that it would be not so easy to envision a blackor-white picture on this subject.

In any case, the major limitation of this paper is represented by the experimental design and the quality of results. For instance, outcomes from the luc-based assays are not so convincing, and the same applies for the RNA analysis. Considering that stable cell lines were used, more robust data would have been obtained by directly analyzing the pattern of both intracellular and supernatant Spike products in WB assays using either readily available polyclonal anti-Spike Abs or upon tagging the N-terminal of transduced Spike sequence. This analysis has also the potential to evaluate the actual stability of possibly truncated Spike products. 
In a similar way, a Northern blot RNA analysis of Spike-expressing cells would be more convincing.

Presented RT-PCR data do not offer a clear picture over the complete pattern of Spike-related RNA transcripts (i.e., a critical result to support the general hypothesis), and lack numerous control conditions.

Finally, both protein and RNA analyses would be reproduced compared to mRNA vaccine-expressing cells.

In conclusion, although the described investigation has an intrinsic paramount relevance, the production of more robust data is necessary to support the interesting hypothesis. 